\title{
Mahlzeiteninsuline
}

\section{Schnell ist noch nicht schnell genug}

—Eine der größten therapeutischen Herausforderungen für Menschen mit Diabetes ist, auch postprandiale Hyperglykämien bestmöglich zu kupieren, betonte Dr. Lena Landstedt-Hallin,

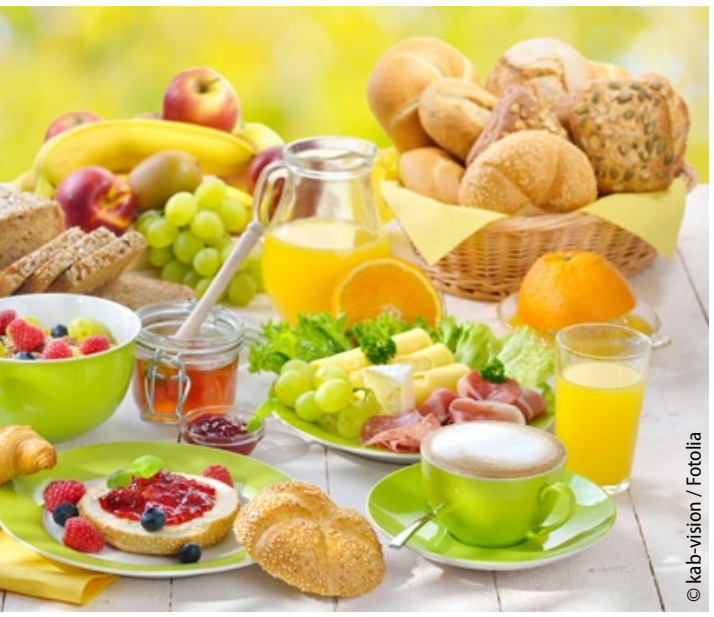

Postprandiale Blutglukosewerte sollten möglichst rasch gesenkt werden.
Stockholm. Schnell wirksame Mahlzeiteninsuline wie Insulin aspart (NovoRapid $^{\star}$ ) haben für insulinpflichtige Menschen mit Diabetes die Situation diesbezüglich zwar verbessert, aber noch nicht optimiert. Ultraschnelle Insuline, die vor allem postprandiale Hyperglykämien kappen und gleichzeitig das Hypoglykämierisiko minimieren, sind wichtige Forschungsziele.

\section{Ein Vitaminmolekül macht den Unterschied}

Faster-acting Insulin aspart, so eine vorläufige Bezeichnung der neuen Insulinaspart-Formulierung, wurde bereits in mehreren klinischen Studien bei Typ-1und insulinpflichtigem Typ-2-Diabetes geprüft. Prof. Keith Bowering, Edmonton, Kanada, verwies auf die Ergebnisse der doppelblinden Phase-IIIa-Studie "onset 1" mit 1.143 Menschen mit Typ-1Diabetes.

\section{Stärkere $\mathrm{HbA}_{1 \mathrm{c}}$-Reduktion}

Im Verlauf von 26 Studienwochen zeigten die schon zuvor optimal auf ein Basalinsulin eingestellten Patienten unter faster-acting Insulin aspart gegenüber der Kontrollgruppe eine signifikant stärkere $\mathrm{HbA}_{1 \mathrm{c}}$-Reduktion. Maßgeblich dafür dürfte sein, dass unter faster-acting Insulin aspart postprandiale Glukosespitzen signifikant stärker gekappt wurden als unter der herkömmlichen Formulierung.

Bei faster aspart kommen neben Insulin aspart die Hilfsstoffe Niacinamid und L-Arginin zur Anwendung. Bezüglich der Nebenwirkungen bestand kein signifikanter Unterschied zwischen den beiden Formulierungen.

Werner Stingl

- Industriesymposium "The Pursuit of ultra-fast Insulins", 52. Jahrestagung der Europäischen Gesellschaft für Diabetesforschung (EASD); München, September 2016 (Veranstalter: Novo Nordisk)

\section{Axilläre Hyperhidrose}

\section{Kurz notiert}

Vitamin- $B_{12}$-Mangel $\rightarrow$ Bei älteren Menschen über 65 Jahren liegt in 10-30\% ein Vitamin- $B_{12}{ }^{-}$ Mangel vor, erläuterte PD Dr. Marija Djukic, Göttingen-Weende. Symptome wie Missempfindungen in den Füßen, ein Manschettengefühl um die Unterschenkel, Unsicherheit auf den Beinen, Sturzneigung sowie die Verschlechterung von Gedächtnis, Stimmung und Alltagskompetenz können Hinweise auf ein Vitamin- $B_{12}$-Defizit sein, erklärte Prof. Karlheinz Reiners, Würzburg. Für die Therapie eignet sich die hochdosierte orale Supplementierung von 1.000-2.000 $\mu \mathrm{g}$ Cyanocobalamin $\left(\mathrm{B}_{12}\right.$ Ankermann $^{\circledast}$ Dragees $1.000 \mu \mathrm{g}$ ), die sich in einer evidenzbasierten Analyse als ebenso effektiv wie die parenterale Applikation erwiesen hat [Vidal-Alaball JV et al. The Cochrane Database Syst Rev. 2005;20,CD004655]. Schwerwiegende und irreversible Folgeschäden können damit vermieden werden.

- 5. Vitamin $B_{12}$-Symposium „Von Gravidität bis GreisenalterVitamin $B_{12}$ für gute Nerven"; Hamburg, April 2016

(Veranstalter: Wörwag)

\section{Methantheliniumbromid ist jetzt erstattungsfähig}

_ Die fokale axilläre oder generalisierte Hyperhidrose belastet die Betroffenen stark. Trotz einer mitunter „sturzbachartigen" Schweißproduktion geht etwas die Hälfte der Betroffenen aus Scham oder aus Unwissenheit nicht zum Arzt [Augustin $M$ et al. Dermatology. 2013;227:10-3], so Prof. Thomas Dirschka, Wuppertal. Oft sei die Hyperhidrose eine Blickdiagnose und Ärzte sollten die Patienten aktiv auf das übermäßige Schwitzen ansprechen, erklärte Dr. Petra Sandow, Berlin. Dermatologen begegnet die Hyperhidrose oft auch in Form von Folgeerkrankungen der erhöhten Hautdurchfeuchtung, etwa als Tinea pedis oder Erythrasma, so Dirschka.

Basis der Zulassung von Methantheliniumbromid (Vagantin ${ }^{\circledR}$ Riemser) war eine Phase-IIIb-Studie mit 339 Teilnehmern [Müller C et al. J Eur Acad Derma- tol Venereol. 2013;10:1278-84], in der Methantheliniumbromid $3 \times 50 \mathrm{mg} / \mathrm{d}$ vs. Placebo signifikant die mittlere axilläre Schweißsekretion verringerte $(\mathrm{p}=$ $0,004)$ und die Lebensqualität auf dem Dermatology Life Quality Index (DLQI) $(\mathrm{p}=0,003)$ und dem Hyperhidrosis Disease Severity Score (HDSS) $(p=0,002)$ verbesserte.

Auch für Frauen im Klimakterium bietet Methantheliniumbromid eine hormonfreie, nicht ZNS-gängige und gut verträgliche Option, betonte Dr. Matthias Krick, Moers. Es sei sehr zu begrüßen, dass Patientinnen die orale Behandlung in Zukunft als erstattungsfähige Option erhalten können.

Dagmar Jäger-Becker

- Pressekonferenz „Neue Aspekte im Therapiemanagement mit Methantheliniumbromid"; Berlin, März 2016 (Veranstalter: Riemser 